\title{
The Harmonic Approximation in Heavy-Ion Reaction Study
}

\author{
Godwin Joseph Ibeh ${ }^{{ }^{*}}$, Elijah Dika Mshelia ${ }^{2}$ \\ ${ }^{1}$ Department of Physics, Nigerian Defence Academy, Kaduna, Nigeria \\ ${ }^{2}$ Department of Physics, University of Abuja, Abuja, Nigeria \\ Email: ${ }^{*}$ gibeh@nda.edu.ng
}

Received 15 August 2015; accepted 13 October 2015; published 16 October 2015

Copyright (C) 2015 by authors and Scientific Research Publishing Inc.

This work is licensed under the Creative Commons Attribution International License (CC BY).

http://creativecommons.org/licenses/by/4.0/

(c) (i) Open Access

\begin{abstract}
The derivation of the harmonic approximation of the Hamiltonian of a model of coupled threedimensional harmonic oscillator is presented. It is shown how the splitting of the total Hamiltonian into the intrinsic and collective Hamiltonians leads to the description of the mechanism for energy dissipation in physical systems.
\end{abstract}

\section{Keywords}

Harmonic Approximation, Energy Dissipation, Coupled Oscillators, Heavy-Ions, Dinuclear System, Cluster Model

\section{Introduction}

Investigation into the mechanism of energy dissipation in heavy-ion reactions has been carried out by different authors from different approaches, an example is the quantum dynamical model of Diaz-Torres, Hinde, Dasgupta, Milburn and Tostevin, which is based on the dissipative dynamics of open quantum systems in this model both deep-inelastic process and quantum tunneling were treated with a quantum mechanical coupled-channels approach [1] and [2]. For some review papers and other approaches to heavy-ion collisions studies, see the following refs. [3]-[11].

Mshelia, Scheid and Greiner formulated a nuclear energy dissipation theory to account for energy dissipation that occurs in heavy-ion collisions [12]. This was described quantum mechanically as resulting from the coupling of collective degrees of freedom to intrinsic excitations. The formalism has been tested on several analytically solvable models of oscillators coupled to free motion in one-dimension [13] [14]. Recently, Ibeh and Mshelia presented a realistic but complex model for investigating the energy dissipation in physical systems [15], which was an extension of the one-dimensional models of previous work [13] [14]. The results obtained showed a

${ }^{*}$ Corresponding author. 
marked improvement to the previous models comparative to the exact results.

In this paper, we consider some of the salient features in the complex model of Ibeh and Mshelia which consists of three-dimensional coupled oscillators located at the corners of a tetrahedron, three oscillators at the corner of the triangular base representing intrinsic motion while the one at the apex represents the collective motion [15]. In Section 2, we present the derivation of the potential energy and the kinetic energy, leading to the equation of motion of the system. Section 3 deals with the quantization of the Hamiltonian of the dissipative system. In Section 4, the solution of the total and intrinsic Schrödinger equations is presented, while Section 5 consists of the method of determining the probability distribution function.

\section{The Classical Hamiltonian}

By the symmetry consideration of the arrangement of four particles in space Figure 1 gives the schematic of the vibrating system, consisting of three-dimensional coupled oscillators located at the corners of a tetrahedronthree oscillators at the corners of the triangular base representing intrinsic motion while one at the apex representing collective motion. All oscillators are coupled to each other elastically. Their equilibrium positions are as follows: the particle of mass $M$ is at the point $(0,0, h)$ at the apex of the tetrahedron while the other three particles are positioned at $\left(-\frac{l}{2},-\frac{l}{2 \sqrt{3}}, 0\right),\left(\frac{l}{2},-\frac{l}{2 \sqrt{3}}, 0\right),\left(0, \frac{l}{\sqrt{3}}, 0\right)$ located at the corners of the triangular base.

For harmonic vibration recall that in classical mechanics [16]-[19], it is shown that near the equilibrium position the potential energy of the system may be developed in a Taylor series i.e.,

$$
2 V=2 V_{0}+2 \sum_{i=1}^{12}\left(\frac{\partial V}{\partial q_{i}}\right)_{0} q_{i}+\sum_{i, j}^{12}\left(\frac{\partial^{2} V}{\partial q_{i} \partial q_{j}}\right) q_{0} q_{j}+\cdots+\text { higher terms }
$$

in this the only term of interest is the third term, which is sufficient for small amplitude of vibration, so that the harmonic potential energy is approximated to

$$
V=\frac{1}{2} \sum_{i, j}^{3 N} V_{i j} q_{i} q_{j}
$$

in which the $V_{i j}$ 's are constants given by

$$
\left(\frac{\partial^{2} V}{\partial q_{i} \partial q_{j}}\right)=V_{i j}=V_{j i}
$$

The quantities $V_{i j}=V_{j i}$ form a symmetric matrix. Thus the potential energy of the system of Figure 1 about the equilibrium positions of the particles, becomes,

$$
\begin{aligned}
2 V= & \frac{3 c}{4 \alpha^{2}}\left\{\left(x_{1}-x\right)^{2}+\frac{1}{3}\left(y_{1}-y\right)^{2}+\frac{4 \beta^{2}}{9}\left(z_{1}-z\right)^{2}+\frac{2 \sqrt{3}}{3}\left(x_{1}-x\right)\left(y_{1}-y\right)\right. \\
& \left.+\frac{4 \beta}{3}\left(x_{1}-x\right) \times\left(z_{1}-z\right)+\frac{4 \beta \sqrt{3}}{9}\left(y_{1}-y\right)\left(z_{1}-z\right)\right\} \\
& +\frac{3 c}{4 \alpha^{2}}\left\{\left(x_{2}-x\right)^{2}+\frac{1}{3}\left(y_{2}-y\right)^{2}+\frac{4 \beta^{2}}{9}\left(z_{2}-z\right)^{2}-\frac{2 \sqrt{3}}{3}\left(x_{2}-x\right)\left(y_{2}-y\right)\right. \\
& \left.-\frac{4 \beta}{3}\left(x_{2}-x\right)\left(z_{2}-z\right)+\frac{4 \beta \sqrt{3}}{9}\left(y_{2}-y\right)\left(z_{2}-z\right)\right\} \\
& +\frac{c}{3 \alpha^{2}}\left\{3\left(y_{3}-y\right)^{2}+\beta^{2}\left(z_{3}-z\right)^{2}-2 \beta \sqrt{3}\left(y_{3}-y\right)^{2}\left(z_{3}-z\right)\right\}+c^{\prime}\left(x_{1}-x_{2}\right) \\
& +\frac{c^{\prime}}{4}\left\{\left(x_{1}-x_{3}\right)^{2}+3\left(y_{1}-y_{3}\right)^{2}+2 \sqrt{3}\left(x_{1}-x_{3}\right)\left(y_{1}-y_{3}\right)\right\} \\
& +\frac{c^{\prime}}{4}\left\{\left(x_{2}-x_{3}\right)^{2}+3\left(y_{2}-y_{3}\right)^{2}-2 \sqrt{3}\left(x_{2}-x_{3}\right)\left(y_{2}-y_{3}\right)\right\}
\end{aligned}
$$




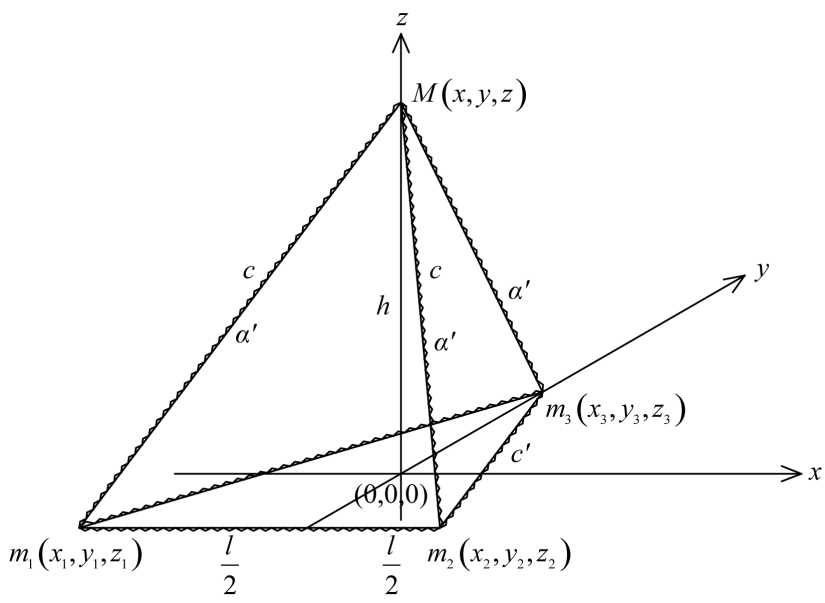

Figure 1. A system of three small mass coupled to each other and each coupled to a large mass.

where the constants, $\beta=\frac{3 h}{l}$ and $\alpha^{2}=\left(1+\frac{\beta^{2}}{3}\right)$.

Similarly the quadratic kinetic energy of the system is

$$
T=\frac{1}{2} m \dot{x}_{1}^{2}+\frac{1}{2} m \dot{y}_{1}^{2}+\frac{1}{2} m \dot{z}_{1}^{2}+\frac{1}{2} m \dot{x}_{2}^{2}+\frac{1}{2} m \dot{y}_{2}^{2}+\frac{1}{2} m \dot{z}_{2}^{2}+\frac{1}{2} m \dot{x}_{3}^{2}+\frac{1}{2} m \dot{y}_{3}^{2}+\frac{1}{2} m \dot{z}_{3}^{2}+\frac{1}{2} M \dot{x}^{2}+\frac{1}{2} M \dot{y}^{2}+\frac{1}{2} M \dot{z}^{2}
$$

where, $\dot{x}_{i}, \dot{y}_{i}, \dot{z}_{i}$ and $\dot{x}, \dot{y}, \dot{z}$ are velocities. $i=1,2,3$.

\section{The Quantum Energy Dissipation}

In Ibeh and Mshelia [15] the quantized Hamiltonian describing the dissipation of energy from the collective motion into intrinsic degrees of freedom is given as:

$$
H=H_{\text {int }}\left(x_{1}, y_{1}, z_{1}, x_{2}, y_{2}, z_{2}, x_{3}, y_{3}, z_{3}, x, y, z\right)+H_{\text {coll }}(x, y, z)
$$

where the intrinsic and collective Hamiltonians are explicitly stated as:

$$
\begin{aligned}
H_{\text {intr }}= & -\frac{\hbar^{2}}{2 m}\left(\frac{\partial^{2}}{\partial x_{1}^{2}}+\frac{\partial^{2}}{\partial y_{1}^{2}}+\frac{\partial^{2}}{\partial z_{1}^{2}}+\frac{\partial^{2}}{\partial x_{2}^{2}}+\frac{\partial^{2}}{\partial y_{2}^{2}}+\frac{\partial^{2}}{\partial z_{2}^{2}}+\frac{\partial^{2}}{\partial x_{3}^{2}}+\frac{\partial^{2}}{\partial y_{3}^{2}}+\frac{\partial^{2}}{\partial z_{3}^{2}}\right) \\
& +\frac{3 c}{8 \alpha^{2}}\left\{\left(x_{1}-x\right)^{2}+\frac{1}{3}\left(y_{1}-y\right)^{2}+\frac{4 \beta^{2}}{9}\left(z_{1}-z\right)^{2}+\frac{2 \sqrt{3}}{3}\left(x_{1}-x\right)\left(y_{1}-y\right)\right. \\
& \left.+\frac{4 \beta}{3}\left(x_{1}-x\right)\left(z_{1}-z\right)+\frac{4 \beta \sqrt{3}}{9} \times\left(y_{1}-y\right)\left(z_{1}-z\right)\right\} \\
& +\frac{3 c}{8 \alpha^{2}}\left\{\left(x_{2}-x\right)^{2}+\frac{1}{3}\left(y_{2}-y\right)^{2}+\frac{4 \beta^{2}}{9}\left(z_{2}-z\right)^{2}-\frac{2 \sqrt{3}}{3}\left(x_{2}-x\right)\right. \\
& \left.\times\left(y_{2}-y\right)-\frac{4 \beta}{3}\left(x_{2}-x\right)\left(z_{2}-z\right)+\frac{4 \beta \sqrt{3}}{9}\left(y_{2}-y\right)\left(z_{2}-z\right)\right\} \\
& +\frac{c}{6 \alpha^{2}}\left\{3\left(y_{3}-y\right)^{2}+\beta^{2} \times\left(z_{3}-z\right)^{2}-2 \beta \sqrt{3}\left(y_{3}-y\right)^{2}\left(z_{3}-z\right)\right\} \\
& +\frac{c^{\prime}}{2}\left(x_{1}-x_{2}\right)+\frac{c^{\prime}}{8}\left\{\left(x_{1}-x_{3}\right)^{2}+3\left(y_{1}-y_{3}\right)^{2}+2 \sqrt{3}\left(x_{1}-x_{3}\right)\left(y_{1}-y_{3}\right)\right\} \\
& +\frac{c^{\prime}}{8}\left\{\left(x_{2}-x_{3}\right)^{2}+3\left(y_{2}-y_{3}\right)^{2}-2 \sqrt{3}\left(x_{2}-x_{3}\right)\left(y_{2}-y_{3}\right)\right\}
\end{aligned}
$$


and

$$
H_{\text {coll }}(x, y, z)=-\frac{\hbar^{2}}{2 M}\left(\frac{\partial^{2}}{\partial x^{2}}+\frac{\partial^{2}}{\partial y^{2}}+\frac{\partial^{2}}{\partial z^{2}}\right)
$$

The collective Hamiltonian is assumed to be that of a free particle with mass, $M$ and described by coordinates $(x, y, z)$. The three oscillators in Figure 1 described by the coordinates $\left(x_{1}, y_{1}, z_{1}, x_{2}, y_{2}, z_{2}, x_{3}, y_{3}, z_{3}\right)$ have the same mass $m$ and are elastically coupled to each other and to the collective motion. Energy can be dissipated from the collective degree of freedom into intrinsic excitations.

\subsection{Normal Modes of Vibration}

The total Hamiltonian in Equation (6) is given in terms of Equations (7) and (8) as

$$
\begin{aligned}
H= & -\frac{\hbar^{2}}{2 m}\left(\frac{\partial^{2}}{\partial x_{1}^{2}}+\frac{\partial^{2}}{\partial y_{1}^{2}}+\frac{\partial^{2}}{\partial z_{1}^{2}}+\frac{\partial^{2}}{\partial x_{2}^{2}}+\frac{\partial^{2}}{\partial y_{2}^{2}}+\frac{\partial^{2}}{\partial z_{2}^{2}}+\frac{\partial^{2}}{\partial x_{3}^{2}}+\frac{\partial^{2}}{\partial y_{3}^{2}}+\frac{\partial^{2}}{\partial z_{3}^{2}}\right)-\frac{\hbar^{2}}{2 M}\left(\frac{\partial^{2}}{\partial x^{2}}+\frac{\partial^{2}}{\partial y^{2}}+\frac{\partial^{2}}{\partial z^{2}}\right) \\
& +\frac{3 c}{8 \alpha^{2}}\left\{\left(x_{1}-x\right)^{2}+\frac{1}{3}\left(y_{1}-y\right)^{2}+\frac{4 \beta^{2}}{9}\left(z_{1}-z\right)^{2}+\frac{2 \sqrt{3}}{3}\left(x_{1}-x\right)\left(y_{1}-y\right)\right. \\
& \left.+\frac{4 \beta}{3}\left(x_{1}-x\right) \times\left(z_{1}-z\right)+\frac{4 \beta \sqrt{3}}{9}\left(y_{1}-y\right)\left(z_{1}-z\right)\right\} \\
& +\frac{3 c}{8 \alpha^{2}}\left\{\left(x_{2}-x\right)^{2}+\frac{1}{3}\left(y_{2}-y\right)^{2}+\frac{4 \beta^{2}}{9}\left(z_{2}-z\right)^{2}-\frac{2 \sqrt{3}}{3}\left(x_{2}-x\right)\left(y_{2}-y\right)\right. \\
& \left.-\frac{4 \beta}{3}\left(x_{2}-x\right)\left(z_{2}-z\right)+\frac{4 \beta \sqrt{3}}{9}\left(y_{2}-y\right)\left(z_{2}-z\right)\right\} \\
& +\frac{c}{6 \alpha^{2}}\left\{3\left(y_{3}-y\right)^{2}+\beta^{2}\left(z_{3}-z\right)^{2}-2 \beta \sqrt{3}\left(y_{3}-y\right)^{2}\left(z_{3}-z\right)\right\}+\frac{c^{\prime}}{2}\left(x_{1}-x_{2}\right) \\
& +\frac{c^{\prime}}{8}\left\{\left(x_{1}-x_{3}\right)^{2}+3\left(y_{1}-y_{3}\right)^{2}+2 \sqrt{3}\left(x_{1}-x_{3}\right)\left(y_{1}-y_{3}\right)\right\} \\
& +\frac{c^{\prime}}{8}\left\{\left(x_{2}-x_{3}\right)^{2}+3\left(y_{2}-y_{3}\right)^{2}-2 \sqrt{3}\left(x_{2}-x_{3}\right) \times\left(y_{2}-y_{3}\right)\right\}
\end{aligned}
$$

From the above consideration we observe that the kinetic energy matrix is diagonal while the potential energy matrix is non-diagonal due to the products $x_{1} x, x_{2} x, y_{1} y, y_{2} y, y_{3} y, z_{1} z, z_{2} z, z_{3} z, x_{1} x_{2}, x_{1} x_{3}$, etc. these off-diagonal terms give rise to the coupling of the collective and intrinsic motions and the coupling of the intrinsic oscillators to each other. By a transformation to normal coordinates the quadratic forms of the kinetic and potential energies in Equation (9), can be reduced simultaneously to sums of squares in these coordinates and their derivatives and hence make the coupled-oscillator problem separable into independent motions, each with a particular normal frequencies [16]-[22].

The normal frequencies are determined by the secular equation

$$
\operatorname{det}\left(V_{i j}-\Omega^{2} T_{i j}\right)=0
$$

where the coefficients $T_{i j}$ and $V_{i j}$ are the elements of the kinetic and potential energy matrices, respectively.

Using the matrices of the kinetic and potential energies according to Equation (9), we obtain from Equation (10) the following twelve eigenfrequencies:

$$
\begin{aligned}
& \Omega_{1}^{2}=\Omega_{3}^{2}=\frac{c\left(B+\sqrt{D^{2}+E}\right)}{12 m \alpha^{2}} ; \quad \Omega_{2}^{2}=\Omega_{4}^{2}=\frac{c\left(B-\sqrt{D^{2}+E}\right)}{12 m \alpha^{2}} ; \quad \Omega_{5}^{2}=\frac{c\left(F+\sqrt{G^{2}+H}\right)}{6 m \alpha^{2}} ; \\
& \Omega_{6}^{2}=\frac{c\left(F-\sqrt{G^{2}+H}\right)}{6 m \alpha^{2}} ; \quad \Omega_{7}^{2}=\Omega_{8}^{2}=\Omega_{9}^{2}=\Omega_{10}^{2}=\Omega_{11}^{2}=\Omega_{12}^{2}=0
\end{aligned}
$$

where, the constants appearing in the eigenfrequencies above are defined as follows: 


$$
\begin{aligned}
& B=6+9 \frac{c^{\prime}}{c} \alpha^{2}+2 \beta^{2}+3 \mu ; \quad D=9 \frac{c^{\prime}}{c} \alpha^{2}-2 \beta^{2}-3 \mu ; E=12\left(3+2 \beta^{2}+3 \mu\right) ; \mu=\frac{3 m}{M} ; \\
& F=3+9 \frac{c^{\prime}}{c} \alpha^{2}+\beta^{2}(\mu+1) ; \quad G=3+9 \frac{c^{\prime}}{c} \alpha^{2}-\beta^{2}(\mu+1) ; H=12 \beta^{2}(\mu+1) .
\end{aligned}
$$

Note the two double degenerate frequencies namely $\Omega_{1}=\Omega_{3}$ and $\Omega_{2}=\Omega_{4}$ and the two non-degenerate eigenfrequencies $\Omega_{5}$ and $\Omega_{6}$ describing the motion in which all the four particles vibrate about their common equilibrium configuration. The six eigenfrequencies: $\Omega_{7} ; \Omega_{8} ; \Omega_{9} ; \Omega_{10} ; \Omega_{11}$ and $\Omega_{12}$ which vanish are assumed to consists of the three zero eigenfrequencies $\Omega_{7}, \Omega_{8}, \Omega_{9}$, corresponding to the eigenmodes describing a uniform translational motion of the system as a whole, while the remaining three zero eigenfrequencies $\Omega_{10}, \Omega_{11}$ and $\Omega_{12}$ have no direct bearing on the theory of energy dissipation in this work. The corresponding transformations to normal coordinates are obtained as:

$$
\begin{aligned}
& g_{1}=\sqrt{-\frac{\left(D-\sqrt{D^{2}+E}\right)}{2\left(3+2 \beta^{2}+3 \mu\right) \sqrt{D^{2}+E}}}\left\{\frac{\left(12+D+\sqrt{D^{2}+E}\right)}{12}\left(x_{1}+x_{2}\right)+\frac{\left(6-D-\sqrt{D^{2}+E}\right)}{6} x_{3}-3 x\right. \\
& \left.+\frac{\left(D+\sqrt{D^{2}+E}\right)}{4 \sqrt{3}}\left(y_{1}-y_{2}\right)-\beta\left(z_{1}-z_{2}\right)\right\} \\
& g_{2}=\sqrt{\frac{D+\sqrt{D^{2}+E}}{2\left(3+2 \beta^{2}+3 \mu\right) \sqrt{D^{2}+E}}}\left\{\frac{\left(-12-D+\sqrt{D^{2}+E}\right)}{12}\left(x_{1}+x_{2}\right)+\frac{\left(-6+D-\sqrt{D^{2}+E}\right)}{6} x_{3}+3 x\right. \\
& \left.-\frac{\left(D-\sqrt{D^{2}+E}\right)}{4 \sqrt{3}}\left(y_{1}-y_{2}\right)-\beta\left(z_{1}-z_{2}\right)\right\} \\
& g_{3}=\sqrt{-\frac{\left(D-\sqrt{D^{2}+E}\right)}{2\left(3+2 \beta^{2}+3 \mu\right) \sqrt{D^{2}+E}}}\left\{\frac{\left(D+\sqrt{D^{2}+E}\right)}{4 \sqrt{3}}\left(x_{1}-x_{2}\right)+\frac{\left(12-D-\sqrt{D^{2}+E}\right)}{12}\left(y_{1}+y_{2}\right)\right. \\
& \left.+\frac{6+D+\sqrt{D^{2}+E}}{6} y_{3}-3 y+\frac{\beta}{\sqrt{3}}\left(z_{1}+z_{2}-2 z_{3}\right)\right\} \\
& g_{4}=\sqrt{-\frac{\left(D-\sqrt{D^{2}+E}\right)}{2\left(3+2 \beta^{2}+3 \mu\right) \sqrt{D^{2}+E}}}\left\{-\frac{\left(D-\sqrt{D^{2}+E}\right)}{4 \sqrt{3}}\left(x_{1}-x_{2}\right)+\frac{\left(-12+D+\sqrt{D^{2}+E}\right)}{12}\left(y_{1}+y_{2}\right)\right. \\
& \left.-\frac{\left(6+D-\sqrt{D^{2}+E}\right)}{6} y_{3}+3 y-\frac{\beta}{\sqrt{3}}\left(z_{1}+z_{2}-2 z_{3}\right)\right\} \\
& g_{5}=\sqrt{-\frac{\left(G-\sqrt{G^{2}+H}\right)}{2(3+3 \mu) \beta^{2} \sqrt{G^{2}+H}}}\left\{\frac{\left(G+\sqrt{G^{2}+H}\right)}{4}\left(x_{1}-x_{2}\right)+\frac{\left(G+\sqrt{G^{2}+H}\right)}{4 \sqrt{3}}\left(y_{1}+y_{2}-2 y_{3}\right)+\beta\left(z_{1}+z_{2}+z_{3}-3 z\right)\right\} \\
& g_{6}=\sqrt{\frac{G+\sqrt{G^{2}+H}}{2(3+3 \mu) \beta^{2} \sqrt{G^{2}+H}}}\left\{-\frac{\left(G-\sqrt{G^{2}+H}\right)}{4}\left(x_{1}-x_{2}\right)-\frac{\left(G+\sqrt{G^{2}+H}\right)}{4 \sqrt{3}}\left(y_{1}+y_{2}-2 y_{3}\right)-\beta\left(z_{1}+z_{2}+z_{3}-3 z\right)\right\} \\
& g_{7}=\frac{1}{(3 m+M)}\left(m x_{1}+m x_{2}+m x_{3}+M x\right) ; \quad g_{8}=\frac{1}{(3 m+M)}\left(m y_{1}+m y_{2}+m y_{3}+M y\right) \\
& g_{9}=\frac{1}{(3 m+M)}\left(m z_{1}+m z_{2}+m z_{3}+M z\right)
\end{aligned}
$$


In terms of the normal coordinates the quantum mechanical total Hamiltonian is

$$
\begin{aligned}
H= & -\frac{\hbar}{2 m} \frac{\partial^{2}}{\partial g_{1}^{2}}-\frac{\hbar}{2 m} \frac{\partial^{2}}{\partial g_{2}^{2}}-\frac{\hbar}{2 m} \frac{\partial^{2}}{\partial g_{3}^{2}}-\frac{\hbar}{2 m} \frac{\partial^{2}}{\partial g_{4}^{2}}-\frac{\hbar}{2 m} \frac{\partial^{2}}{\partial g_{5}^{2}}-\frac{\hbar}{2 m} \frac{\partial^{2}}{\partial g_{6}^{2}}-\frac{\hbar}{2(3 m+M)} \frac{\partial^{2}}{\partial g_{7}^{2}}-\frac{\hbar}{2(3 m+M)} \frac{\partial^{2}}{\partial g_{8}^{2}} \\
& -\frac{\hbar}{2(3 m+M)} \frac{\partial^{2}}{\partial g_{9}^{2}}+\frac{1}{2} m\left(\Omega_{1}^{2} g_{1}^{2}+\Omega_{2}^{2} g_{2}^{2}+\Omega_{3}^{2} g_{3}^{2}+\Omega_{4}^{2} g_{4}^{2}+\Omega_{5}^{2} g_{5}^{2}+\Omega_{6}^{2} g_{6}^{2}\right)
\end{aligned}
$$

\subsection{The Solutions of the Schrödinger Equation for the Total and Intrinsic Hamiltonians}

We now obtain solutions of the time-independent Schrödinger equation with the decoupled total Hamiltonian $H$ given by Equation (13).

$$
H \Psi\left(g_{1}, g_{2}, \cdots, g_{9}\right)=E \Psi\left(g_{1}, g_{2}, \cdots, g_{9}\right)
$$

Since $H$ describes a free translational motion of the centre of mass and the decoupled harmonic oscillators in the $g_{1}, g_{2}, g_{3}, g_{4}, g_{5}$ and $g_{6}$ degrees of freedom the eigenvalues and eigenfunctions are obtained as,

$$
E_{k^{\prime} n_{1} n_{2} n_{3} n_{4} n_{5} n_{6}}=\frac{\hbar^{2}}{2(3 m+M)}\left(k_{x}^{2}+k_{y}^{2}+k_{z}^{2}\right)+\sum_{i=1}^{6} \hbar \Omega_{i}\left(n_{i}+\frac{1}{2}\right)
$$

and

$$
\Psi_{k_{x} k_{y} k_{z} n_{1} n_{2} n_{3} n_{4} n_{5} n_{6}}\left(g_{1}, g_{2}, \cdots, g_{9}\right)=U_{k_{x}}\left(g_{7}\right) U_{k_{y}}\left(g_{8}\right) U_{k_{z}}\left(g_{9}\right) \prod_{i=1}^{6} \psi_{n_{i}}\left(g_{i}\right)
$$

where, the quantum numbers are $n_{1}, n_{2}, n_{3}, n_{4}, n_{5}, n_{6}=0,1,2, \cdots$. and $k_{x}, k_{y}, k_{z}$ are the wave numbers of the plane-wave functions for the centre of mass, normalized by means of the Dirac $\delta$-function [23] [24] are given by

$$
\begin{aligned}
& U_{k_{x}}\left(g_{7}\right)=\frac{1}{\sqrt{2 \pi}} \exp \left[i k_{x} g_{7}\right] ; \\
& U_{k_{y}}\left(g_{8}\right)=\frac{1}{\sqrt{2 \pi}} \exp \left[i k_{y} g_{8}\right] ; \\
& U_{k_{z}}\left(g_{9}\right)=\frac{1}{\sqrt{2 \pi}} \exp \left[i k_{z} g_{9}\right],
\end{aligned}
$$

The normalized, bound state, wave functions of the harmonic oscillators are written as

$$
\psi_{n_{i}}\left(g_{i}\right)=N_{n_{i}} \exp \left[-\frac{1}{2} \lambda_{i}^{2} g_{i}^{2}\right] H_{n_{i}}\left(\lambda_{i} g_{i}\right), i=1,2, \cdots, 6
$$

The quantity $H_{n_{i}}$ is a Hermite polynomial of order $n_{i}$ and the $\lambda_{i}$ s are the inverse oscillator lengths given by

$$
\lambda_{i}=\left(\frac{m \Omega_{i}}{\hbar}\right)^{\frac{1}{2}}
$$

the normalization constant $N_{n_{i}}$ occurring in Equation (19) is defined by

$$
N_{n_{i}}=\left(\frac{\lambda_{i}}{\pi^{\frac{1}{2}} 2^{n_{i}} n_{i} !}\right)^{\frac{1}{2}} .
$$

The total wave function in Equation (16) is normalized as follows:

$$
\int \cdots \int \Psi_{k_{x} k_{y} k_{z} n_{1} n_{2} n_{3} n_{4} n_{5} n_{6}}^{*} \Psi_{k_{x} k_{y} k_{z} n_{1} n_{2} n_{3} n_{4} n_{5} n_{6}} \mathrm{~d} g_{1} \cdots \mathrm{d} g_{9}=\delta\left(k_{x}-k_{x}^{\prime}\right) \cdots \delta\left(k_{z}-k_{z}^{\prime}\right) \delta_{n_{1} n_{1}^{\prime}} \cdots \delta_{n_{6} n_{6}^{\prime}}
$$

The intrinsic Hamiltonian can be stated in terms of intrinsic coordinates defined as following 


$$
\begin{aligned}
& \xi_{1}=\left(x+\frac{\sqrt{3}}{3} y+\frac{2 \beta}{3} z\right)+\zeta_{1} ; \quad \zeta_{1}=\left(x_{1}-x\right)+\frac{\sqrt{3}}{3}\left(y_{1}-y\right)+\frac{2 \beta}{3}\left(z_{1}-z\right) \\
& \xi_{2}=\left(x-\frac{\sqrt{3}}{3} y-\frac{2 \beta}{3} z\right)+\zeta_{2} ; \quad \zeta_{2}=\left(x_{2}-x\right)-\frac{\sqrt{3}}{3}\left(y_{2}-y\right)-\frac{2 \beta}{3}\left(z_{2}-z\right) \\
& \xi_{3}=(\sqrt{3} y-\beta z)+\zeta_{3} ; \quad \zeta_{3}=\sqrt{3}\left(y_{3}-y\right)-\beta\left(z_{3}-z\right) \\
& \xi_{4}=\left(x_{1}-x_{2}\right) ; \\
& \xi_{5}=\left(x_{1}-x_{3}\right)+\sqrt{3}\left(y_{1}-y_{3}\right) ; \\
& \xi_{6}=\left(x_{2}-x_{3}\right)-\sqrt{3}\left(y_{2}-y_{3}\right) .
\end{aligned}
$$

The resulting eigenvalue equation of the intrinsic Hamiltonian is

$$
\sum_{i=1}^{6}\left(-\frac{\hbar^{2}}{2 m} \frac{\partial^{2}}{\partial r_{i}^{2}}+\frac{1}{2} m \omega_{i}^{2} r_{i}^{2}\right) \Phi_{t_{i}}\left(r_{1} r_{2} \cdots r_{6}\right)=\varepsilon \Phi_{t_{i}}\left(r_{1} r_{2} \cdots r_{6}\right)
$$

where $r_{i}$ are the intrinsic normal coordinates defined in terms of the intrinsic coordinates given in Equation (22).

Solving Equation (23) results in the eigenvalues

$$
\varepsilon=\sum_{i=1}^{6} \hbar \omega_{i}\left(t_{i}+\frac{1}{2}\right), \quad t_{i}=0,1,2, \cdots
$$

And the following set of eigenfrequencies and eigenfunctions:

$$
\begin{aligned}
& \omega_{1}^{2}=\omega_{3}^{2}=\frac{c\left(b+\sqrt{d^{2}+e}\right)}{12 m \alpha^{2}} ; \\
& \omega_{2}^{2}=\omega_{4}^{2}=\frac{c\left(b-\sqrt{d^{2}+e}\right)}{12 m \alpha^{2}} ; \\
& \omega_{5}^{2}=\frac{c\left(f+\sqrt{g^{2}+h}\right)}{6 m \alpha^{2}} ; \\
& \omega_{6}^{2}=\frac{c\left(f-\sqrt{g^{2}+h}\right)}{6 m \alpha^{2}}
\end{aligned}
$$

where, the constants appearing in the eigenfrequencies above are defined as follows:

$$
\begin{aligned}
& b=6+9 \frac{c^{\prime}}{c} \alpha^{2}+2 \beta^{2} ; \quad d=9 \frac{c^{\prime}}{c} \alpha^{2}-2 \beta^{2} ; \quad e=12\left(3+2 \beta^{2}\right) ; \\
& f=3+9 \frac{c^{\prime}}{c} \alpha^{2}+\beta^{2} ; \quad h=12 \beta^{2} ; \quad g=3+9 \frac{c^{\prime}}{c} \alpha^{2}-\beta^{2} .
\end{aligned}
$$

the normalized intrinsic oscillator eigenfunctions:

$$
\varphi_{t_{i}}\left(r_{i}\right)=\eta_{t_{i}} \exp \left[-\frac{1}{2} \gamma_{i}^{2} r_{i}^{2}\right] H_{t_{i}}\left(\gamma_{i} r_{i}\right),
$$

where, the intrinsic inverse oscillator lengths and normalization constants are respectively,

$$
\gamma_{i}=\left(\frac{m \omega_{i}}{\hbar}\right)^{\frac{1}{2}},
$$




$$
\eta_{t_{i}}=\left(\frac{\gamma_{i}}{\pi^{\frac{1}{2}} 2^{t_{i}} t_{i} !}\right)^{\frac{1}{2}}, \quad t_{i}=0,1,2,3, \cdots ; i=1,2,3, \cdots, 6
$$

From Equation (26) the total intrinsic wave-function becomes

$$
\Phi_{t_{i}}\left(r_{1}, r_{2}, \cdots, r_{6}\right)=\varphi_{t_{1}}\left(r_{1}\right) \varphi_{t_{2}}\left(r_{2}\right) \varphi_{t_{3}}\left(r_{3}\right) \varphi_{t_{4}}\left(r_{4}\right) \varphi_{t_{5}}\left(r_{5}\right) \varphi_{t_{6}}\left(r_{6}\right)
$$

\section{The Probability Density Functions}

The fact that the intrinsic Hamiltonian eigenfunctions obtained form a complete set, by use of the completeness relation the total wave function $\Psi_{k_{x} k_{y} k_{z} n_{1} n_{2} n_{3} n_{4} n_{5} n_{6}}\left(\xi_{1}, \xi_{2}, \cdots, \xi_{6}, x, y, z\right)$ is expanded in terms of the complete orthonormal set of oscillator functions $\Phi_{t_{1} t_{2} t_{4} t_{4} t_{5} t_{6}}\left(\xi_{1}, \xi_{2}, \cdots, \xi_{6}, x, y, z\right)$. The normalization of $\Psi_{k_{x} k_{y} k_{z} n_{1} n_{2} n_{3} n_{4} n_{5} n_{6}}$ gives

$$
\begin{aligned}
\int_{-\infty}^{\infty} \Psi_{\theta}^{*} \Psi_{\theta} \mathrm{d} \xi_{1} \mathrm{~d} \xi_{2} \mathrm{~d} \xi_{3} \mathrm{~d} \xi_{4} \mathrm{~d} \xi_{5} \mathrm{~d} \xi_{6} & =\sum_{i} \sum_{j} f_{i}^{*}(x, y, z) f_{j}(x, y, z) \iint_{-\infty}^{\infty} \Phi_{i}^{*} \Phi_{j} \mathrm{~d} \xi_{1} \mathrm{~d} \xi_{2} \mathrm{~d} \xi_{3} \mathrm{~d} \xi_{4} \mathrm{~d} \xi_{5} \mathrm{~d} \xi_{6} \\
& =\sum_{i}\left|f_{i}(x, y, z)\right|^{2}
\end{aligned}
$$

since the $\Phi_{i}$ 's are orthonormal. The expansion coefficients $f_{i}(x, y, z)$ represent the probability amplitude for excitation of the intrinsic motion [25].

On the other hand, the normalization of $\Psi_{k_{x} k_{y} k_{z} n_{1} n_{2} n_{3} n_{4} n_{5} n_{6}}\left(\xi_{1}, \xi_{2}, \cdots, \xi_{6}, x, y, z\right)$ with respect to the variables $g_{1}$, $g_{2}, g_{3}, g_{4}, g_{5}$ and $g_{6}$ gives

$$
\begin{aligned}
& \iint_{-\infty}^{\infty} \Psi_{\theta}^{*} \Psi_{\theta} \mathrm{d} g_{1} \mathrm{~d} g_{2} \mathrm{~d} g_{3} \mathrm{~d} g_{4} \mathrm{~d} g_{5} \mathrm{~d} g_{6} \\
& =\psi_{k_{x}}^{*}\left(g_{7}\right) \psi_{k_{x}}\left(g_{7}\right) \psi_{k_{y}}^{*}\left(g_{8}\right) \psi_{k_{y}}\left(g_{8}\right) \psi_{k_{z}}^{*}\left(g_{9}\right) \psi_{k_{z}}\left(g_{9}\right) \int_{-\infty}^{\infty} \psi_{n_{1}}^{*}\left(g_{1}\right) \psi_{n_{1}}\left(g_{1}\right) \mathrm{d} g_{1} \\
& \quad \times \int_{-\infty}^{\infty} \psi_{n_{2}}^{*}\left(g_{2}\right) \psi_{n_{2}}\left(g_{2}\right) \mathrm{d} g_{2} \int_{-\infty}^{\infty} \psi_{n_{3}}^{*}\left(g_{3}\right) \psi_{n_{3}}\left(g_{3}\right) \mathrm{d} g_{3} \int_{-\infty}^{\infty} \psi_{n_{4}}^{*}\left(g_{4}\right) \psi_{n_{4}}\left(g_{4}\right) \mathrm{d} g_{4} \\
& \quad \times \int_{-\infty}^{\infty} \psi_{n_{5}}^{*}\left(g_{5}\right) \psi_{n_{5}}\left(g_{5}\right) \mathrm{d} g_{5} \int_{-\infty}^{\infty} \psi_{n_{6}}^{*}\left(g_{6}\right) \psi_{n_{6}}\left(g_{6}\right) \mathrm{d} g_{6}=\frac{1}{8 \pi^{3}}
\end{aligned}
$$

The relationship between the left hand and the right hand of Equations (30) and (31) is given by the transformation

$$
\begin{aligned}
& \iint_{-\infty}^{\infty} \Psi_{\theta}^{*}\left(g_{1}, g_{2}, g_{3}, g_{4}, g_{5}, g_{6}, g_{7}, g_{8}, g_{9}\right) \Psi_{\theta}\left(g_{1}, g_{2}, g_{3}, g_{4}, g_{5}, g_{6}, g_{7}, g_{8}, g_{9}\right) \mathrm{d} g_{1} \mathrm{~d} g_{2} \mathrm{~d} g_{3} \mathrm{~d} g_{4} \mathrm{~d} g_{5} \mathrm{~d} g_{6} \\
& =\iint_{-\infty}^{\infty} \Psi_{\theta}^{*}\left(\xi_{1}, \xi_{2}, \xi_{3}, \xi_{4}, \xi_{5}, \xi_{6}, x, y, z\right) \Psi_{\theta}\left(\xi_{1}, \xi_{2}, \xi_{3}, \xi_{4}, \xi_{5}, \xi_{6}, x, y, z\right)\left|\frac{\partial\left(g_{1}, g_{2}, g_{3}, g_{4}, g_{5}, g_{6}\right)}{\partial\left(\xi_{1}, \xi_{2}, \xi_{3}, \xi_{4}, \xi_{5}, \xi_{6}\right)}\right| \mathrm{d} \xi_{1} \mathrm{~d} \xi_{2} \mathrm{~d} \xi_{3} \mathrm{~d} \xi_{4} \mathrm{~d} \xi_{5} \mathrm{~d} \xi_{6}
\end{aligned}
$$

When values are substituted the Jacobian is

$$
\left|\frac{\partial\left(g_{1}, g_{2}, g_{3}, g_{4}, g_{5}, g_{6}\right)}{\partial\left(\xi_{1}, \xi_{2}, \xi_{3}, \xi_{4}, \xi_{5}, \xi_{6}\right)}\right|=1
$$

Comparing Equations (31), (32) and (33) the normalization condition

$$
\sum_{n_{1}, n_{2}, n_{3}, n_{4}, n_{5}, n_{6}}\left|f_{t_{1}, t_{2}, t_{3}, t_{4}, t_{5}, t_{6}}^{n_{2}, r_{2}, n_{6}, n_{6}}(x, y, z)\right|^{2}=\sum_{t_{1}, t_{2}, t_{3}, t_{4}, t_{5}, t_{6}}\left|f_{t_{1}, t_{2}, t_{3}, t_{4}, t_{5}, t_{6}}^{n_{2}, n_{2}, n_{6}, n_{6}}(x, y, z)\right|^{2}=\frac{1}{8 \pi^{3}}
$$

Equation (34) gives a measure for the probability for intrinsic excitation from collective motion [15]. It should 
be noted that the derivation of Equation (34) demonstrates that the form of the collective amplitude, $f_{t_{1}, t_{2}, t_{3}, n_{4}, t_{5}, t_{5}}^{n_{1}, n_{5}, n_{6}, n_{6}}(x, y, z)$ satisfy the normalization condition for the total wave function.

The collective amplitude is the expansion coefficient of the total wave function

$\Psi_{k_{x} k_{y} k_{z} n_{1} n_{2} n_{3} n_{4} n_{5} n_{6}}\left(\xi_{1}, \xi_{2}, \cdots, \xi_{6}, x, y, z\right)$ when expanded in terms of the complete orthonormal set of oscillator functions $\Phi_{t_{1} t_{2} t_{4} t_{5} t_{6} t_{6}}\left(\xi_{1}, \xi_{2}, \cdots, \xi_{6}, x, y, z\right)$, and its form can be obtained by using Equations (12), (16), (17), (18) and

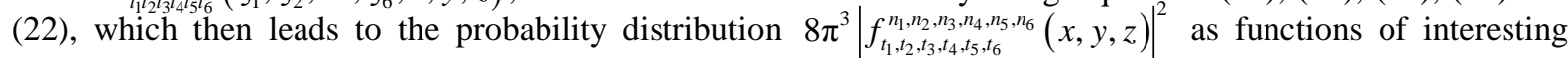
physical parameters for example, energy, intrinsic and collective quantum numbers, etc.

\section{Conclusions}

This work has shown that the harmonic approximation of the Hamiltonian of coupled oscillators leads to a Schrödinger equation which describes the coupling of collective degree of freedom, represented by free motion with intrinsic degrees of freedom, represented by three coupled oscillators. This model explains the mechanism for energy dissipation in a physical system, based on the coupling of intrinsic and collective degrees of freedom. The model can be extended to nuclear fission and heavy-ion reactions, where the collective degree of freedom is the relative coordinates of the two heavy-ions and the intrinsic degrees of freedom are the single-particle degrees of freedom [22].

Furthermore, of current interest and one which is an extension of the above model is the cluster model consisting of a dinuclear system which is not easily solvable analytically because it includes other degrees of freedoms such as butterfly, belly-dancer-type motions, $\gamma$-and $\beta$-vibrations, etc., of individual nuclei, this model is based on the assumption that cluster-type shapes are produced in the mass asymmetry of nuclear molecules. Theoretical and experimental evidences exist that show that this model is capable of explaining many of the features of deformed heavy nuclei [26]-[38]. An example of such features is the resent work of Adamian, Antonenko and Lenske, in which the linear response theory was used to calculate the mass parameters for collective variables of the dinuclear systems formed in cold fusion reactions and found that the microscopic mass parameter in the neck is larger than the one obtained using the hydrodynamical model [39].

\section{References}

[1] Diaz-Torres, A., Hinde, D.J., Dasgupta, M., Milburn, G.J. and Tostevin, J.A. (2008) Dissipative Quantum Dynamics in Low-Energy Collisions of Complex Nuclei. Physical Review C, 78, Article ID: 064604. http://dx.doi.org/10.1103/physrevc.78.064604

[2] Diaz-Torres, A., Hinde, D.J., Dasgupta, M., Milburn, G.J. and Tostevin, J.A. (2009) Coupled-Channels Approach for Dissipative Quantum Dynamics in Near-Barrier Collisions. International Conference on New Aspects of Heavy-Ion Barrier, Chicago. http://dx.doi.org/10.1063/1.3108859

[3] Swiatecki, W.J. and Bjфrnholm, S. (1972) Fission and Fusion Dynamics. Physics Reports, 4, 326-342. http://dx.doi.org/10.1016/0370-1573(72)90003-8

[4] Nörenberg, W. and Weidenmüller, H.A. (1976) Introduction to the Theory of Heavy-Ion Collisions. Lecture Notes in Physics 5, Springer-Verlag, Berlin.

[5] Hasse, R.W. (1978) Approaches to Nuclear Friction. Reports on Progress in Physics, 41, 1027-1101. http://dx.doi.org/10.1088/0034-4885/41/7/002

[6] Weidenmüller, H.A. (1980) Transport Theories of Heavy-Ion Reactions. Progress in Particle and Nuclear Physics, 3 , 49. http://dx.doi.org/10.1016/0146-6410(80)90030-7

[7] Mshelia, E.D. (1997) Nuclear Science and Technology in Human Progress. (Unpublished University Inaugural Lecture). Abubakar Tafawa Balewa University, Bauchi.

[8] Aritomo, Y., Hagino, K., Nishio, K. and Chiba, S. (2012) Dynamical Approach to Heavy-Ion Induced Fission Using Actinide Target Nuclei at Energies around the Coulomb Barrier. Physical Review C, 85, Article ID: 044614. http://dx.doi.org/10.1103/PhysRevC.85.044614

[9] Liu, Z.H. and Bao, J.D. (2013) Possibility to Produce Element 120 in the 54Cr+248Cm Hot Fusion Reaction. Physical Review C, 87, Article ID: 0344616. http://dx.doi.org/10.1103/PhysRevC.87.034616

[10] Gontchar, I.I., Bhattacharya, R. and Chushnyakova, M.V. (2014) Quantitative Analysis of Precise Heavy-Ion Fusion Data at Above-Barrier Energies Using Skyrme-Hartree-Fock Nuclear Densities. Physical Review C, 89, Article ID: 034601. http://dx.doi.org/10.1103/PhysRevC.89.034601

[11] Wen, K., Sakata, F., Li, Z.-X., Wu, X., Zhang, Y. and Zhou, S. (2014) Energy Dependence of the Nucleus-Nucleus 
Potential and the Friction Parameter in Fusion Reactions. Physical Review C, 90, Article ID: 054613. http://dx.doi.org/10.1103/PhysRevC.90.054613

[12] Mshelia, E.D., Scheid, W. and Greiner, W. (1975) Theory of Energy Dissipation in Heavy-Ion Reactions. Il Nuovo Cimento A, 30, 589-608. http://dx.doi.org/10.1007/BF02730488

[13] Mshelia, E.D., Haln, D. and Scheid, W. (1981) Energy Dissipation in a Model of Coupled Oscillators. Il Nuovo Cimento A, 61, 28-55. http://dx.doi.org/10.1007/BF02776606

[14] Mshelia, E.D. and Ngadda, Y.H. (1989) A Stimulation for Energy Dissipation in Nuclear Reactions. Journal of Physics G: Nuclear and Particle Physics, 15, 1281-1290. http://dx.doi.org/10.1088/0954-3899/15/8/023

[15] Ibeh, G.J. and Mshelia, E.D. (2014) Energy Dissipation in a Model of Coupled Three-Dimensional Harmonic Oscillator. Far East Journal of Mathematical Sciences, 88, 107-136.

[16] Corben, H.C. and Stehle, P. (1960) Classical Mechanics. John Wiley, New York, 113-131, 364-372.

[17] Wells, D.A. (1967) Theory and Problems of Lagrangian Dynamics. Schaum Publication, New York.

[18] Marion, J.B. and Thornton, S.T. (1995) Classical Dynamics of Particles and Systems. 4th Edition, Saunders College Publications, New York.

[19] Goldstein, H., Poole, C. and Safko, J. (2002) Classical Mechanics. 3rd Edition, Addison-Wesley, San Francisco, 238258.

[20] Wilson Jr., B., Decius, J.C. and Paul, C.C. (1980) Molecular Vibrations. Dover Publications, Inc., New York.

[21] Desloge, E.A. (1982) Classical Mechanics. Volume II, Wiley-Interscience, New York, 665-707.

[22] Mshelia, E.D. (1995) Method of Normal Coordinates in the Formulation of a System with Dissipation: The Harmonic Oscillator. Il Nuovo Cimento A, 108, 709-721. http://dx.doi.org/10.1007/BF02813376

[23] Schiff, L.I. (1987) Quantum Mechanics. 3rd Edition, McGraw-Hill, New York, 50-52.

[24] Greiner, W. (1994) Quantum Mechanics: An Introduction. 3rd Edition, Springer-Verlag, New York, 97-105.

[25] Courant, R. and Hilbert, D. (1953) Methods of Mathematical Physics. Interscience Publishers, New York, 424.

[26] Bromley, D.A. (1978) Nuclear Molecules. Scientific American, 239, 58-68. http://dx.doi.org/10.1038/scientificamerican1278-58

[27] Volkov, V.V. (1978) Deep Inelastic Transfer Reaction-The New Type of Reactions between Complex Nuclei. Physics Reports, 44, 93-157. http://dx.doi.org/10.1016/0370-1573(78)90200-4

[28] Iachello, F. and Jackson, A.D. (1982) A Phenomenological Approach to $\alpha$-Clustering in Heavy Nuclei. Physics Letters B, 108, 151-154. http://dx.doi.org/10.1016/0370-2693(82)91162-5

[29] Greiner, W., Park, J.Y. and Scheid, W. (1995) Nuclear Molecules. World Scientific, Singapore.

[30] Buck, B., Merchant, A.C. and Perez, S.M. (1998) Systematic Study of Exotic Clustering in Even-Even Actinide Nuclei. Physical Review C, 58, 2049-2060. http://dx.doi.org/10.1103/physrevc.58.2049

[31] Buck, B., Merchant, A.C. and Perez, S.M. (1999) Cluster Structure and Gamma Transitions in Actinides. Physical Review C, 59, 750-754. http://dx.doi.org/10.1103/PhysRevC.59.750

[32] Adamian, G.G., Antonenko, N.V. and Scheid, W. (2000) Isotopic Dependence of Fusion Cross Sections in Reactions with Heavy Nuclei. Nuclear Physics A, 678, 24-38. http://dx.doi.org/10.1016/s0375-9474(00)00317-1

[33] Mshelia, E.D. and Scheid, W. (2004) Collective Dynamics of a Dinuclear System. The European Physical Journal A, 20, 251-254. http://dx.doi.org/10.1140/epja/i2003-10102-7

[34] Li, W., Nan, W., Fei, J., Hushan, X., Wei, Z., Li, Q.F., et al. (2006) Particle Transfer and Fusion Cross-Section for Super-Heavy Nuclei in Dinuclear System. Journal of Physics G, 32, 1143-1155. http://dx.doi.org/10.1088/0954-3899/32/8/006

[35] Kalandarov, S.A., Adamian, G.G., Antonenko, N.V. and Scheid, W. (2011) Role of Angular Momentum in the Production of Complex Fragments in Fusion and Quasifission Reactions. Physical Review C, 83, Article ID: 054611. http://dx.doi.org/10.1103/PhysRevC.83.054611

[36] Kalandarov, S.A., Adamian, G.G, Antonenko, N.V., Scheid, W., Heinz, S., Comas, V., Hofman, S., Khuyagbaatar, J., Ackermann, D., Heredia, J., Heßberger, F.P., Kindler, B., Lommel, B. and Mann, R. (2011) Emission of Cluster with Z > 2 from Excited Actinide Nuclei. Physical Review C, 84, Article ID: 054607. http://dx.doi.org/10.1103/PhysRevC.84.054607

[37] Kalandarov, S.A., Adamian, G.G., Antonenko, N.V., Scheid, W. and Wieleczko, J.P. (2011) Role of the Entrance Channel in the Production of Complex Fragments in Fusion-Fission and Quasifission Reactions in the Framework of the Dinuclear System Model. Physical Review C, 84, Article ID: 064601.

http://dx.doi.org/10.1103/PhysRevC.84.064601 
[38] Bansal, M., Sahila, C. and Rajk, G. (2012) Dynamical Cluster-Decay Model Using Various Formulations of a Proximity Potential for Compact Non-Coplanar Nuclei: Application to the ${ }^{64} \mathrm{Ni}+{ }^{100}$ Mo Reaction. Physical Review C, 86, Article ID: 034604. http://dx.doi.org/10.1103/physrevc.86.034604

[39] Adamian, G.G., Antonenko, N.V. and Lenske, H. (2015) Role of the Neck Degree of Freedom in Cold Fusion Reactions. Physical Review C, 91, Article ID: 054602. http://dx.doi.org/10.1103/PhysRevC.91.054602 